\title{
Unión biliopancreática anómala. Una enfermedad no exclusiva de pacientes orientales
}

\author{
J. GARCÍA CANO, M. A. GODOY ${ }^{1}$, J. MORILLAS ARIÑO, J. I. PÉREZ GARCÍA \\ Sección de Aparato Digestivo. 'Servicio de Radiología. Hospital Virgen de la Luz. Cuenca
}

\section{RESUMEN}

La union biliopancreática anómala se produce cuando el conducto biliar y pancreático se unen fuera de la pared duodenal, sin que parezca existir el mecanismo esfinteriano del Oddi. Se ha pensado que esta es una enfermedad predominantemente de pacientes orientales. Sin embargo, cada vez se hacen más diagnósticos en otros grupos poblacionales. Presentamos el caso de una mujer de 41 años con dolor epigástrico intermitente y elevación leve de la amilasa. El diagnóstico no se pudo realizar por medio de una colangiopancreatografía por resonancia magnética, quizás por falta de experiencia. Se diagnosticó por medio de una colangiopancreatografía retrógrada endoscópica. En ella se apreció una unión biliopancreática anómala de ángulo agudo, con una unión compleja entre el colédoco y el conducto pancreático. Se observó también una dilatación fusiforme de la vía biliar y del hepático principal izquierdo. Se remitió a la paciente para una resección del conducto biliar y un procedimiento derivativo quirúrgico. Sin embargo, quizás también por falta de experiencia, se decidió el seguimiento periódico de la paciente. En conclusión, la unión biliopancreática anómala se diagnostica también en poblaciones occidentales y se precisa más experiencia en su diagnóstico y tratamiento.

PALABRAS CLAVE: Unión biliopancreática anómala. Quistes coledocianos. Epigastralgia crónica. CPRE.
PANCREATOBILIARY MALJUNCTION. NOT ONLY AN EASTERN DISEASE

\begin{abstract}
Pancreatobiliary maljunction, an extramural junction of the pancreatic and biliary ducts in the duodenum, apparently beyond the scope of intramural sphincter function, is thought to be a preferential disease of Eastern people. However, this diagnosis is increasingly being made in other populations. We present a 41-year-old woman who presented with intermittent epigastric pain and mild raise in amylase levels. MRCP did not offer a definitive diagnosis, perhaps because of lack of experience in this disease. Finally, ERCP showed a pancreatobiliary maljunction, type acute angle, but with a complex connection between the choledocus and the pancreatic duct. Fusiform dilation of the common bile duct and of the left main hepatic duct were also present. The patient was referred for extrahepatic biliary resection and a diversion procedure. But also because of lack of experience in this disease, a wait-and-see approach was undertaken. In conclusion, pancreatobiliary maljunction will be increasingly diagnosed in western communities and more diagnostic and therapeutic experience will be needed.
\end{abstract}

KEY WORDS: Pancreatobiliary maljunction. Choledochal cysts. Chronic epigastric pain. ERCP.

García Cano J, Godoy MA, Morillas Ariño J, Pérez García JI. Unión biliopancreática anómala. Una enfermedad no exclusiva de pacientes orientales. An Med Interna (Madrid) 2007; 24: 384-386.

\section{INTRODUCCIÓN}

La unión biliopancreática anómala (UBPA) es un trastorno congénito en el que el colédoco y el Wirsung se unen antes de insertarse en la pared duodenal (Fig. 1). Se forma así un largo canal común biliopancreático (mayor de $1 \mathrm{~cm}$ ), en el que ambos conductos no están recubiertos por las fibras musculares del esfínter de Oddi. Al no funcionar el mecanismo esfinteriano, las secreciones biliar y pancreática se mezclan (1).

En muchas ocasiones la UBPA produce quistes biliares que presentan un riesgo elevado de malignización. Aún en los casos en que la vía biliar no está dilatada, los pacientes con UBPA tienen mayor frecuencia de neoplasias de la vesícula biliar.

La mayoría de pacientes descritos con UBPA son orientales. Sin embargo, la facilidad de acceso a los recursos sanitarios y la generalización de las pruebas de imagen, hacen que cada vez se diagnostiquen más casos en otros grupos raciales y poblacionales (2).

Presentamos el caso de una paciente de nuestro medio con UBPA y quistes biliares asociados.

Trabajo aceptado: 15 de marzo de 2007 


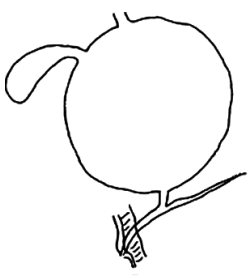

Tipo A

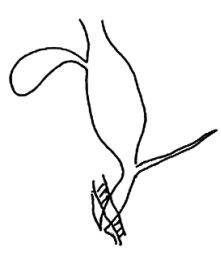

Tipo B

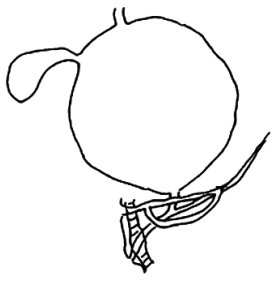

Tipo C
Fig. 1. Tipos de union biliopancreática anómala (UBPA). En el tipo A el colédoco parece desembocar en el Wirsung, por eso se le conoce también como tipo C-P o unión en ángulo recto. En el tipo B, el páncreas parece desembocar en el colédoco, se le conoce también como tipo P-C o en ángulo agudo. En el tipo C la unión es compleja. En todos los casos se observa el largo canal común biliopancreático que se forma fuera de la pared duodenal y, que, por lo tanto, no dispone del mecanismo esfinteriano del Oddi.

\section{CASO APORTADO}

Mujer de 41 años que consultó por dolor epigástrico intermitente con elevación leve de la amilasa sérica. Tras un estudio exhaustivo, se decidió realizar una colangiopancreatografía por resonancia magnética (CPRM). El diagnóstico no fue concluyente aunque se observó una dilatación del colédoco y del hepático izquierdo. Se decidió entonces realizar una Colangiopancreatografía retrógrada encoscópica (CPRE) para intentar clarificar este hallazgo. La papila de Vater presentaba un aspecto endoscópico normal. Tras canular la papila se introdujo contraste para opacificar los conductos. Se observó (Fig. 2A) una dilatación quística fusiforme del colédoco y otro quiste en el conducto hepático principal izquierdo. El colédoco distal se unía al pancreas ventral a una distancia de $1,75 \mathrm{~cm}$. desde el orificio papilar, produciéndose así el llamado canal común de las UBPA. Se pasó una guía al colédoco y se inyectó contraste en otro punto del orificio papilar. Se vio que el canal común se unía al páncreas dorsal a nivel de la papila Minor, por medio de una pequeña rama anterior del páncreas ventral. Se observó también salida de bilis por la papila Minor, sugiriendo una comunicación del páncreas dorsal con el colédoco. Al inyectar contraste por la papila Minor (Fig. 2B), se observó una comunicación en forma de asa entre el páncreas dorsal y el páncreas ventral del canal común. Al finalizar la CPRE se dejaron dos prótesis plásticas a través de la papila de Vater. Una de ellas en el colédoco para evitar una posible colangitis y otra en el páncreas. No obstante, la paciente presentó una pancreatitis leve, de la que se recuperó satisfactoriamente. Se remitió a la paciente a un cirujano experto en la vía biliar para valorar la realización de una exéresis de los quistes, que implicaba la resección de la vía biliar extrahepática. Sin embargo, el consejo quirúrgico fue el seguimiento periódico de la paciente. Desde hace dos años, la enferma sigue presentando algún dolor epigástrico esporádico inespecífico y las colangiopancreatografías por resonancia magnética no han mostrado cambios sustanciales.

\section{DISCUSIÓN}

El caso aportado es un ejemplo de que la UBPA no es una enfermedad exclusiva de pacientes orientales. Nuestra enferma es de raza blanca, al igual que toda su familia, que ha vivido siempre en el entorno de nuestra provincia.

El diagnóstico y el tratamiento de este trastorno, junto con los quistes biliares asociados en esta paciente adulta, ha pre-

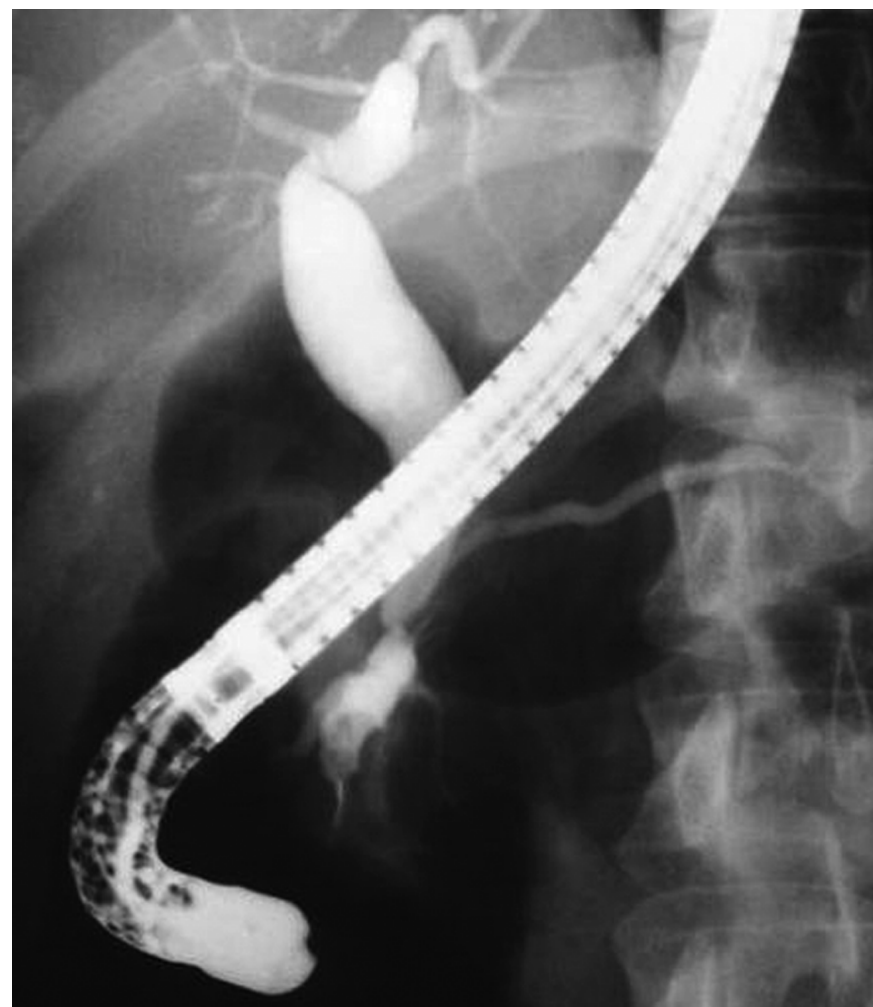

Fig. 2A. A la izquierda, se observa la inyección de contraste desde el orificio papilar. La unión biliopancreática anómala parece corresponder a un tipo $B$, aunque, vista con más detalle, tiene características también del tipo C. En la colangiografía se aprecia un quiste fusiforme en el colédoco. Se observa otro quiste en el hepático principal izquierdo. El colédoco distal se une con el conducto pancreático ventral a los 1,75 cm desde la papila, formando el canal común.

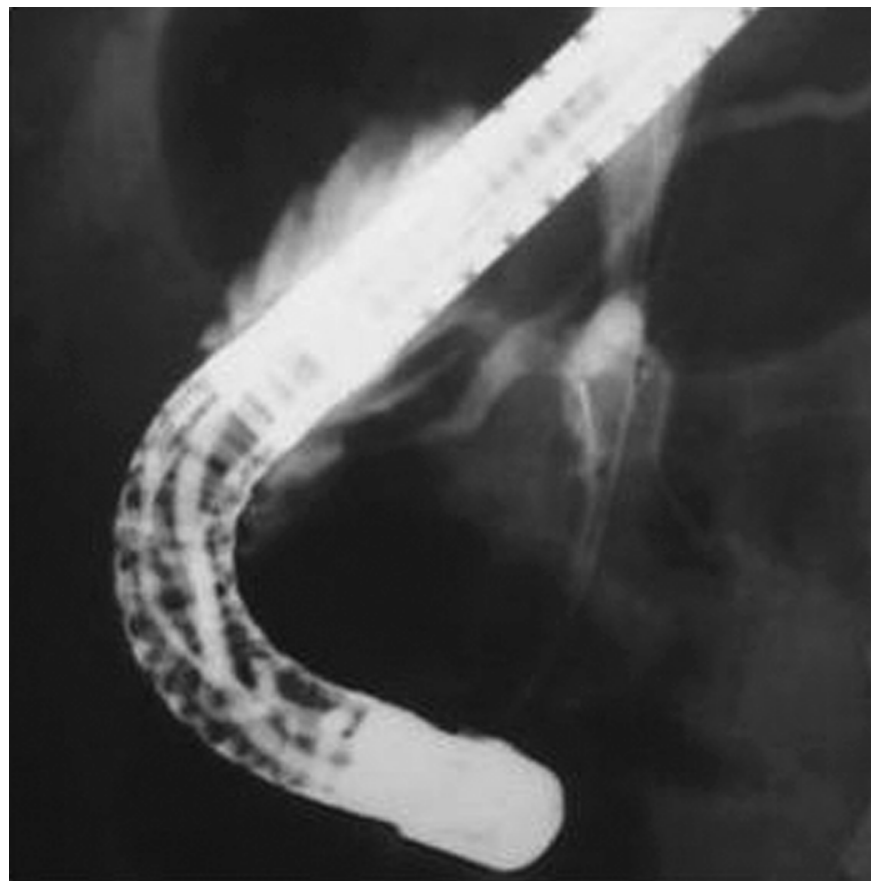

Fig. 2B. A la derecha, se ha canulado la papila Minor. Con la inyección de contraste en el páncreas dorsal se observa un asa que comunica este conducto dorsal con la rama anterior del páncreas ventral. En esta imagen se comprueba que el tipo de unión biliopancreática anómala de este caso tiene características del tipo C (ver figura 1). Se rellena también con contraste el colédoco. 
sentado ciertas dificultades que pensamos son de importancia clínica. En primer lugar, la rareza en nuestro medio de esta afección hizo que el estudio de la vía biliar por CPRM no fuera concluyente. En el momento actual, la CPRM puede sustituir a la CPRE en la valoración inicial de la UBPA y de los quistes biliares (3). Aunque para el tipo III de los quistes biliares o coledococele y para pequeños cambios ductales de la UBPA, sea preciso recurrir a la CPRE (4).

El inyectar contraste en una cavidad quística, como se hace en la CPRE, puede conllevar una colangitis si no se garantiza el drenaje adecuado. En nuestro caso, no se produjo porque dejamos una pequeña prótesis plástica que se retiró varias semanas después. Del mismo modo, la inyección de contraste en el Wirsung y la manipulación de la papila de Vater pueden producir pancreatitis, como ocurrió en nuestra paciente, aunque fue leve (5).

Nuestra paciente presentaba un tipo B de UBPA o de ángulo agudo (Fig. 1), aunque la unión entre el colédoco y los conductos pancreáticos es compleja -tipo C-. El quiste biliar fusiforme asociado puede encuadrarse en el tipo IVa (dilata-

\section{Bibliografía}

1. The Japanese Study Group on Pancreaticobiliary Maljunction. Diagnostic criteria of pancreatico-biliary maljunction. J Hepatobiliary Pancreat Surg 1994; 1: 219-21

2. Adham M, Valette PJ, Partensky. Pancreaticobiliary maljunction without choledochal dilatation associated with gallbladder cancer: report of 2 European cases. Surgery 2005; 138: 961.

3. Park DH, Kim MH, Lee SK, Lee SS, Choi JS, Lee YS, et al. Can MRCP replace the diagnostic role of ERCP for patients with choledochal cysts? Gastrointest Endosc 2005; 62: 360-6.

4. García Cano J, Nieto J, Godoy MA, Gómez-Ruiz CJ, Sánchez-Manja- ción quística fusiforme del colédoco y de ambos conductos hepáticos principales), aunque, en este caso, sólo había otro quiste en el hepático principal izquierdo.

La rareza de esta enfermedad en nuestro medio, no sólo ocasionó dificultades en el diagnóstico mediante CPRM, sino, también en el tratamiento. En los casos de UBPA con dilatación biliar se debe realizar una exéresis de toda la vía biliar extrahepática dilatada (6). El criterio no es tan firme si la UBPA no se une a dilatación del colédoco, aunque en estos pacientes hay una alta incidencia de cánceres de vesícula biliar. A pesar de que la paciente fue remitida a un grupo quirúrgico experto en cirugía biliar, este tratamiento pareció muy agresivo para una paciente joven y prácticamente asintomática. Sin embargo, es el tratamiento de elección para los cirujanos que tratan numerosos casos de UBPA y quistes biliares (6).

Como conclusión, es posible que cada vez se diagnostiquen más pacientes con UBPA y, por lo tanto, sería preciso aumentar la experiencia en el diagnóstico y tratamiento de esta enfermedad. vacas N, Pérez Sola A. Coledococele de pequeño tamaño como causa de dolor abdominal crónico: diagnóstico y tratamiento mediante CPRE. An Med Interna (Madrid) 2005; 22: 591-3.

5. García Cano Lizcano J, González Martín JA, Morillas Ariño J, Pérez Sola A. Complicaciones de la colangiopancreatografía retrógrada endoscópica. Estudio en una unidad pequeña de CPRE. Rev Esp Enferm Dig 2004; 96: 163-73.

6. Okada A, Hasegawa T, Oguchi Y, Nakamura T. Recent advances in pathophysiology and surgical treatment of congenital dilation of the bile duct. J Hepatobiliary Pancreat Surg 2002; 9: 342-5. 